\title{
Observation of hard scattering in photoproduction at HERA
}

\author{
ZEUS Collaboration
}

M. Derrick, D. Krakauer, S. Magill, B. Musgrave, J. Repond, K. Sugano ${ }^{1}$, R. Stanek, R.L. Talaga , J. Thron

Argonne National Laboratory, Argonne, IL 60439. USA

F. Arzarello, R. Ayed ${ }^{2}$, G. Barbagli ${ }^{3}$, G. Bari, M. Basile, L. Bellagamba, D. Boscherini, G. Bruni, P. Bruni, G. Cara Romeo, G. Castellini ${ }^{4}$, M. Chiarini, L. Cifarelli, F. Cindolo, F. Ciralli, A. Contin, S. D'Auria, C. Del Papa, F. Frasconi, P. Giusti, G. Iacobucci, G. Laurenti, G. Levi, Q. Lin, B. Lisowski, G. Maccarrone, A. Margotti, T. Massam, R. Nania . C. Nemoz, F. Palmonari, G. Sartorelli, R. Timellini, Y. Zamora Garcia ${ }^{2}$, A. Zichichi University of Bologna and INFN Bologna, 1-40126 Bologna, Italy

A. Bargende, F. Barreiro ${ }^{5}$, J. Crittenden, H. Dabbous, K. Desch, B. Diekmann, M. Geerts, G. Geitz, B. Gutjahr, H. Hartmann, J. Hartmann, D. Haun, K. Heinloth, E. Hilger, H.-P. Jakob, S. Kramarczyk, M. Kückes, A. Mass, S. Mengel, J. Mollen, H. Müsch, E. Paul, R. Schattevoy, B. Schneider ${ }^{6}$, J.-L. Schneider, R. Wedemeyer

Physikalisches Institut der Universität Bonn, W-5300 Bonn I, FRG

A. Cassidy, D.G. Cussans, N. Dyce, H.F. Fawcett, B. Foster, R. Gilmore, G.P. Heath, M. Lancaster, T.J. Llewellyn, J. Malos, C.J.S. Morgado, R.J. Tapper, S.S. Wilson

Bristol University, Bristol BS\& ITL, UK

R.R. Rau

Brookhaven National Laboratory, Upton, NY 11973, LSA

A. Bernstein, A. Caldwell, I. Gialas, J.A. Parsons, S. Ritz, F. Sciulli ${ }^{7}$, P.B. Straub, L. Wai , S. Yang

Nevis Laboratories, Columbia University. Irvington on Hudson. NY 10533, LSA

T. Barillari, M. Schioppa, G. Susinno

Physics Department, Calabria University, and INFN, I-87036 Cosenza, Italy

W. Burkot, J. Chwastowski ${ }^{8}$, A. Dwuraźny, A. Eskreys, B. Niziol, Z. Jakubowski ${ }^{9}$, K. Piotrzkowski, M. Zachara, L. Zawiejski

Institute of Nuclear Physics, Cracow, Poland 
P. Borzemski, K. Eskreys, K. Jeleń, D. Kisielewska, T. Kowalski, J. Kulka, E. Rulikowska-Zarębska, L. Suszycki, J. Zając

Institute of Physics and Nuclear Techniques, Academy of Mining and Metallurgy, Cracow, Poland

T. Kędzierski , A. Kotański, M. Przybycień

Department of Physics, Jagellonian L'niversity, PL-30059 Cracow, Poland

L.A.T. Bauerdick, U. Behrens, J.K. Bienlein, C. Coldewey, A. Dannemann, K. Dierks ${ }^{10}$, W. Dorth ${ }^{10}$, G. Drews, P. Erhard ${ }^{11}$, M. Flasiński ${ }^{12}$. I. Fleck, A. Fürtjes, R. Gläser ${ }^{13}$, P. Göttlicher, T. Haas, L. Hagge, W. Hain, D. Hasell, H. Hultschig, G. Jahnen ${ }^{14}$, P. Joos, M. Kasemann, R. Klanner, W. Koch, U. Kötz, H. Kowalski, J. Labs, A. Ladage , B. Löhr, M. Löwe, D. Lüke, J. Mainusch, O. Manczak ${ }^{15}$, M. Momayezi . S. Nickel, D. Notz, I. Park, K.-U. Pösnecker ${ }^{16}$, M. Rohde, E. Ros ${ }^{8}$, U. Schneekloth, J. Schroeder, W. Schulz, F. Selonke , E. Tscheslog ${ }^{17}$, T. Tsurugai , F. Turkot ${ }^{18}$, W. Vogel ${ }^{19}$. T. Woeniger ${ }^{20}$, G. Wolf, C. Youngman Deutsches Elektronen-Synchrotron DESY, W-2000 Hamburg 52, FRG

H.J. Grabosch, A. Leich, A. Meyer, C. Rethfeldt, S. Schlenstedt Institut für Hochenergiephysik, DESY-Zeuthen, O-1615 Zeuthen, FRG

R. Casalbuoni, S. De Curtis, D. Dominici, A. Francescato, M. Nuti, P. Pelfer, University of Florence and INFN, I-50I25 Florence, Ilaly.

G. Anzivino, R. Casaccia, I. Laakso ${ }^{21}$, S. De Pasquale, S. Qian, L. Votano INFN, Laboratori Nazionali di Frascati. I-00044 Frascati, Italy

A. Bamberger, A. Freidhof, T. Poser, S. Söldner-Rembold, G. Theisen, T. Trefzger Physikalisches Institut der Universitat Freiburg, W-7800 Freiburg, FRG

N.H. Brook, P.J. Bussey, A.T. Doyle, J.R. Forbes, V.A. Jamieson, C. Raine, D.H. Saxon Department of Physics and Astronomy. University of Glasgow, Glasgow G12 8QQ. L'K

G. Gloth, U. Holm, H. Kammerlocher, B. Krebs, T. Neumann, K. Wick I. Institute of Experimental Physics. Hamburg University. $W$-2000 Ilamburg. FRG

A. Hofmann ${ }^{21}$, W. Kröger, J. Krüger, E. Lohrmann, J. Milewski ${ }^{15}$, M. Nakahata, N. Pavel, G. Poelz, R. Salomon ${ }^{22}$, A. Seidman ${ }^{23}$, W. Schott , B.H. Wiik, F. Zetsche

II. Institute of Experimental Physics, Hamburg Liniversity, $\boldsymbol{W}$-2000 Hamburg 50, FRG

T.C. Bacon, I. Butterworth, C. Markou, D. McQuillan, D.B. Miller, M.M. Mobayyen , A. Prinias, A. Vorvolakos Migh Energy Nuclear Physics Group, Imperial College London. London SW7 2BZ, LK 
T. Bienz, H. Kreutzmann, U. Mallik, E. McCliment, M. Roco, M.Z. Wang

Physics and Astronomy Department, University of Iowa, Iowa City, IA 52240. USA

P. Cloth, D. Filges

Institut für Kernphysik, Forschungszentrum Jülich, W-5170 Jülich 1. FRG

L. Chen, R. Imlay, S. Kartik, H.-J. Kim, R.R. McNeil, W. Metcalf

Department of Physics and Astronomy, Louisiana State University, Baton Rouge, LA 70803, USA

G. Cases, L. Hervás ${ }^{24}$, L. Labarga ${ }^{24}$, J. del Peso, J. Roldán, J. Terrón, J.F. de Trocóniz ${ }^{25}$

Departamento de Física Teórica, Universidad Autónoma de Madrid, E-28049 Madrid, Spain

F. Ikraiam, J.K. Mayer, G.R. Smith

Department of Physics, University of Manitoba, Winnipeg, Manitoba, Canada R3T 2N2

F. Corriveau, D.J. Gilkinson, D.S. Hanna ', L.W. Hung, J.W. Mitchell, P.M. Patel, L.E. Sinclair, D.G. Stairs, R. Ullmann

Department of Physics, McGill University, Montreal. Quebec. Canada H3A 2T8

G.L. Bashindzhagyan, P.F. Ermolov, Y.A. Golubkov, V.A. Kuzmin, E.N. Kuznetsov, A.A. Savin, A.G. Voronin, N.P. Zotov

Institute of Nuclear Pysics, Moscow State University, 117234 Moscow, Russian Federation

S. Bentvelsen, A. Dake, J. Engelen, P. de Jong, S. de Jong ${ }^{8}$, M. de Kamps, P. Kooijman, A. Kruse, H. van der Lugt, V. O'Dell, J. Straver ${ }^{8}$, A. Tenner, H. Tiecke, H. Uijterwaal ${ }^{26}$, J. Vermeulen, L. Wiggers, E. de Wolf, R. van Woudenberg, R. Yoshida NIKHEF, NL-1009 DB Amsterdam. The Netherlands

B. Bylsma, L.S. Durkin, C. Li, T.Y. Ling, K.W. McLean, W.N. Murray, S.K. Park, T.A. Romanowski, R. Seidlein

Physics Department, Ohio State University, Columbus, OH 43210, LSA

G.A. Blair, J.M. Butterworth, A. Byrne, R.J. Cashmore, A.M. Cooper-Sarkar, R.C.E. Devenish, D.M. Gingrich, P.M. Hallam-Baker ${ }^{9}$, N. Harnew, T. Khatri, K.R. Long, P. Luffman, I. McArthur, P. Morawitz, J. Nash, S.J.P. Smith ${ }^{27}$, N.C. Roocroft , F.F. Wilson Department of Physics, University of Oxford, Oxford, $U K$

G. Abbiendi, R. Brugnera, R. Carlin, F. Dal Corso, M. De Giorgi, U. Dosselli, C. Fanin, F. Gasparini, S. Limentani, M. Morandin, M. Posocco, L. Stanco, R. Stroili, C. Voci

Dipartimento di Fisica dell' Università and INFN, 1-35131 Padua, Italy 
J.N. Lim , B.Y. Oh ${ }^{28}$. J. Whitmore ${ }^{29}$

Department of Physics, Pennsylvania State Liniversity, L'niversity Park. PA 16802, USA

M. Bonori . U. Contino . G. D’Agostini, M. Guida, M. Iori , S. Mari, G. Marini , M. Mattioli , D. Monaldi, A. Nigro

Dipartimento di Fisica. L'niversità "La Sapienza". I-00185 Rome, Italy'

J.C. Hart, N.A. McCubbin, T.P. Shah, T.L. Short

Rutherford Appleton Laboratory; Chilton. Didcot, Oxon OXII OQX, LK

E. Barberis , N. Cartiglia , C. Heusch, B. Hubbard, J. Leslie, J.S.T. Ng, K. O'Shaughnessy, H.F. Sadrozinski, A. Seiden

Liniversity of California. Santa Cruz. CA 95064. USA

E. Badura, J. Biltzinger, H. Chaves, M. Rost, R.J. Seifert, A.H. Walenta , W. Weihs . G. Zech Fachbereich Physik der Universität-Gesamihochschule Siegen, W'-5900 Siegen 21, FRG

S. Dagan. R. Heifetz, A. Levy, D. Zer-Zion

School of Physics, Tel-Aviv Liniversity. Tel Avir 69978, Israel

T. Hasegawa, M. Hazumi, T. Ishii . S. Kasai ${ }^{30}$, M. Kuze, Y. Nagasawa, M. Nakao, H. Okuno, K. Tokushuku, T. Watanabe, S. Yamada

Inslitute for Nuclear Study, University of Tokyo, Tokyo 188. Japan

M. Chiba, R. Hamatsu, T. Hirose, S. Kitamura, S. Nagayama, Y. Nakamitsu

Department of Physics, Tokyo Metropolitan L'niversity. Tokyo 158. Japan

M. Arneodo, M. Costa, M.I. Ferrero, L. Lamberti, S. Maselli, C. Peroni, A. Solano, A. Staiano Dipartimento di Fisica Sperimentale, Lniversità di Torino, and INFN, 1-10125 Turin. Italy

M. Dardo

Facultá di Scienze MFN, Liniversità di Torino, Alessandria, Italy

D.C. Bailey, D. Bandyopadhyay, F. Benard, S. Bhadra, M. Brkic, B.D. Burow, F.S. Chlebana, M.B. Crombie, G.F. Hartner, G.M. Levman, J.F. Martin, R.S. Orr, J.D. Prentice, C.R. Sampson, G.G. Stairs, R.J. Teuscher, T.-S. Yoon

Department of Physics, Liniversity of Toronto, Toronto, Ontario, Canada MSS $1 \mathrm{~A} 7$

F.W. Bullock, C.D. Catterall, J.C. Giddings, T.W. Jones, A.M. Khan, J.B. Lane , P.L. Makkar, D. Shaw, J. Shulman

Physics and Astronomy Department, Lniversity College London, London HCIE 6BT, UK 
K. Blankenship, J. Kochocki, B. Lu, L.W. Mo

Physics Department. Virginia Polytechnic Institute, Blacksburg, VA 24061, USA

K. Charchuła, J. Ciborowski, J. Gajewski, G. Grzelak, M. Kasprzak, M. Krzyżanowski , K. Muchorowski , R.J. Nowak, J.M. Pawlak, K. Stojda, A. Stopczyński, R. Szwed, T. Tymieniecka ${ }^{31}$, R. Walczak, A.K. Wróblewski, J.A. Zakrzewski, A.F. Zarnecki

Institute of Experimental Physics, Warsaw University, PL-00681 Warsaw, Poland

\title{
M. Adamus
}

Institute for Nuclear Studies, Warsaw, Poland

\author{
H. Abramowicz ${ }^{15}$, Y. Eisenberg, C. Glasman, U. Karshon, A. Montag, D. Revel, A. Shapira \\ Nuclear Physics Department, Weizmann Institute, Rehovot 76100, Israel
}
I. Ali, B. Behrens, U. Camerini, S. Dasu , C. Fordham, C. Foudas, A. Goussiou, M. Lomperski, R.J. Loveless, P. Nylander, M. Ptacek, D.D. Reeder, W.H. Smith, S. Silverstein

Department of Physics, Lniversity of Wisconsin, Madison, WI 53706, LSA

\author{
W.R. Frisken, K.M. Furutani and Y. Iga \\ Department of Physics, York Liniversity, North York, Ontario, Canada
}

Received 19 October 1992

\begin{abstract}
We report a study of electron proton collisions at very low $Q^{2}$, corresponding to virtual photoproduction at centre of mass energies in the range $100-295 \mathrm{GeV}$. The distribution in transverse energy of the observed hadrons is much harder than can be explained by soft processes. Some of the events show back-to-back two-jet production at the rate and with the characteristics expected from hard two-body scattering. A subset of the two-jet events have energy in the electron direction consistent with that expected from the photon remnant in resolved photon processes.
\end{abstract}

Present address: UC Santa Cruz, Santa Cruz, CA, USA. Supported by Worldlab, Lausanne, Switzerland. Present address: INFN, Florence, Italy.

Also at IROE, Florence, Italy.

Alexander von Humboldt Fellow, on leave of absence from Universidad Autónoma de Madrid, Madrid, Spain. Present address: INFAS, Bonn, FRG.

Present address: DESY, Hamburg, FRG, as Alexander von Humboldt Fellow.

Present address: CERN, Geneva, Switzerland.

Present address: DESY, Hamburg, FRG.

Now a self-employed consultant.

Present address: IST GmbH, FRG.

On leave from Jagellonian University, Cracow, Poland.

Present address: Martin \& Associates, Hamburg, FRG.

Present address: Harry Hoffmann, Fitzbek, FRG.

On leave from Warsaw University; Warsaw, Poland.

Present address: Lufthansa, Frankfurt, FRG.

Present address: Integrata, Frankfurt, FRG.
13 On leave from FERMILAB, Batavia, IL, USA

19 Present address: Blohm \& Voss, Hamburg, FRG.

20 Present address: BMW, Munich, FRG.

21 Deceased.

22 Left II. Institute of Experimental Physics, University of Hamburg, in 1990.

23 On leave from Tel Aviv University, Tel Aviv, Israel.

21 Partially supported by Comunidad Autónoma de Madrid, Spain.

25 Supported by Fundación Banco Exterior.

26 Present address: SSC, Dallas, TX, USA.

27 Now with McKinsey Consultants, Sidney, Australia.

28 On leave and supported by DESY 1992-93.

29 On leave and supported by DESY 1991-92.

30 Present address: Hiroshima National College of Maritime Technology, Hiroshima, Japan.

31 Presently on leave of absence at NIKHEF Amsterdam, The Netherlands. 


\section{Introduction}

Deep inelastic lepton-proton scattering (DIS) is traditionally understood to proceed via the exchange of a neutral or charged boson, which interacts with a quark in the proton. At lowest order in perturbation theory, the final state consists of the scattered lepton, the jet associated with the struck quark, and the proton remnant. Since the proton remnant takes very little transverse momentum $\left(p_{1}\right)$, the scattered lepton and the quark jet balance $p_{t}$. At high momentum transfer squared $Q^{2}$, two-jet production appears as a higher order correction, and is understood as due to gluon radiation from the struck quark.

In contrast, at large centre of mass energy and small values of $Q^{2}$, hard collisions between quasireal photons and partons from the proton give rise to two or more partons in the final state. In these photoproduction processes, the electron is scattered at very small angles, and the parton jets balance $p_{1}$. The photon can interact with a gluon, yielding a $q \vec{q}$ pair (boson-gluon fusion) [1], or with a quark, generating a $q g$ final state (QCD Compton scattering) [2]. These are known as direct photon processes.

If the exchanged photon is quasi-real, the interaction is also sensitive to its internal structure. Thus, one has to consider the possibility that a parton in the photon interacts with a parton in the proton. These are known as resolved photon processes [3].

Calculations have been made of the relative contributions of the various parton-parton and photonparton processes at HERA energies [4]. They depend strongly on the minimum transverse momentum $p_{\text {tmin }}$ involved in the two-body scattering process, as well as on the structure functions used, in particular that of the photon. The cross-section for hard photoproduction is dominated by the resolved photon processes.

Evidence for hard photon scattering, in quantitative agreement with QCD calculations, has been found in two-photon physics at $e^{+} e^{-}$colliders [5], as well as in fixed target expcriments [6-8], but the restricted phase space available at the energies of these experiments has not allowed the observation of two-jet production. Here we present evidence both for jets in hard photon-proton scattering and for resolved photon processes, using the ZEUS detector at HERA ${ }^{* 1}$.

\section{HERA machine conditions}

Currently at HERA, bunches of electrons with energy $E_{e}=26.6 \mathrm{GeV}$ collide with bunches of protons of energy $820 \mathrm{GeV}$ with a time between bunch crossings of $96 \mathrm{~ns}$. HERA is designed to run with 210 bunches in each of the electron and proton rings. For the data taking period described in this paper only ten consecutive bunches (0-9) were filled for each beam. In addition electron bunch number 19 was filled for measuring electron induced background. For approximately half of the runs, the electron bunch number 9 was left empty, in order to facilitate proton background studies. The electron and proton beam currents were typically 1-2 mA. The length of the interaction region, determined by the proton bunch length, was about $40 \mathrm{~cm}$.

\section{Experimental setup}

ZEUS is a multipurpose magnetic detector $[10,11]$, as shown in fig. 1. Here we give a brief description concentrating on those parts of the detector relevant for the present analysis.

Charged particles are tracked by the inner tracking detectors. These surround the beam pipe and are operated in a magnetic field of $1.43 \mathrm{~T}$ provided by a thin superconducting coil. The central tracking detector consists of 72 cylindrical drift chamber layers, organised into 9 "superlayers" [12]. At present the tracks are reconstructed using a subset of layers equipped with z-by-timing electronics $\# 2$ which compares the arrival times at both ends of the chamber. The resolution is $4.0 \mathrm{~cm}$ in $z$ and about $1 \mathrm{~mm}$ in the $x y$ plane.

The solenoid is surrounded by a high resolution calorimeter divided into three sections, for-

* 1 Preliminary results on hard photoproduction obtained with the $\mathrm{Hl}$ detector were reported in ref. [9].

\#2 The ZEUS coordinate system is defined as right handed with the $z$ axis pointing in the proton beam direction, hereafter referred to as forward, and the $x$ axis pointing to the centre of HERA. 


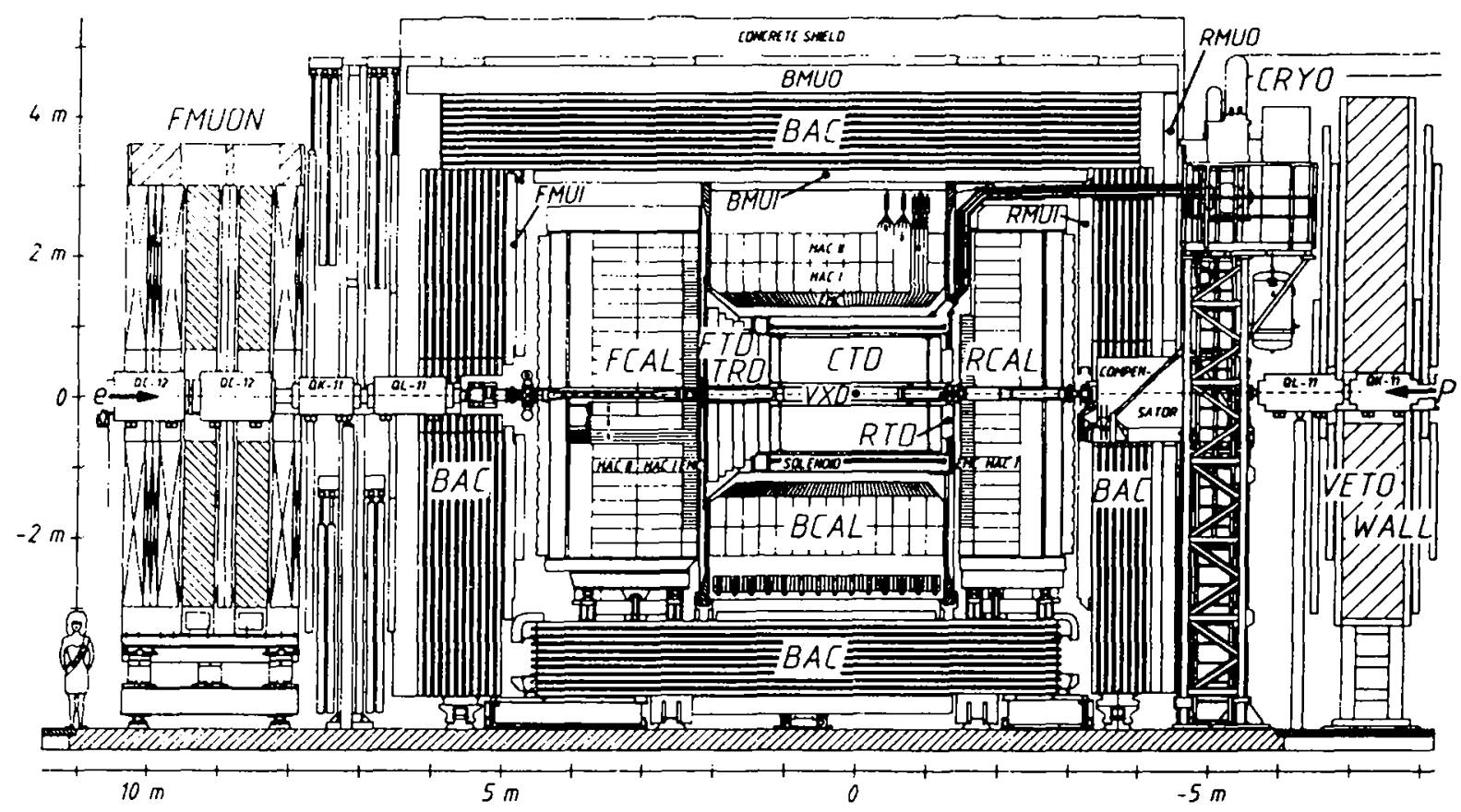

Fig. 1. Side view of the ZEUS detector.

ward (FCAL), barrel (BCAL) and rear (RCAL). The calorimeter is built with alternating layers of depleted uranium and scintillator with one radiation length sampling. Longitudinally the calorimeter is segmented into electromagnetic (EMC) and hadronic (HAC) sections. The calorimeter is read out via wavelength shifter bars and photomultipliers. The transverse cell sizes are about $5 \mathrm{~cm} \times 20 \mathrm{~cm}$ for the FCAL and BCAL EMC, $10 \mathrm{~cm} \times 20 \mathrm{~cm}$ for the RCAL EMC, $20 \mathrm{~cm} \times 20 \mathrm{~cm}$ for the FCAL and RCAL HAC, and up to $25 \mathrm{~cm} \times 35 \mathrm{~cm}$ for the BCAL HAC. Under test beam conditions the energy resolution for electrons was found to be $\sigma(E) / E=0.18 / \sqrt{E}$ ( $E$ in $\mathrm{GeV}$ ) and for hadrons $\sigma(E) / E=0.35 / \sqrt{E}$ $[13,14]$. The time resolution of a calorimeter cell is $1.5 / \sqrt{E} \oplus 0.5 \mathrm{~ns}[13,15]$.

In order to discriminate against the very high interaction rate produced upstream of the detector by interactions of the protons with residual gas in the vacuum pipe (beam-gas), two layers of scintillator counters are installed on either side of an $87 \mathrm{~cm}$ thick iron wall (VETOWALL). In addition a scintillator lead sandwich counter (C5) partially surrounds the beam pipe just upstream of the rear calorimeter.
To measure the luminosity as well as to tag very small $Q^{2}$ processes, we use two lead scintillator electromagnetic calorimeters [16]. Hard bremsstrahlung photons emerging from the electron-proton interaction point (IP) at angles $\theta_{\gamma} \leqslant 0.5 \mathrm{mrad}$ with respect to the electron beam axis hit the photon calorimeter at $107 \mathrm{~m}$ from the IP. Electrons emitted from the IP at scattering angles $\theta_{c}^{\prime} \leqslant 6 \mathrm{mrad}$ and with energies $0.2 E_{e}<E_{\rho}^{\prime}<0.9 E_{e}$ arc deflected by beam magnets and hit the electron calorimeter placed $34.7 \mathrm{~m}$ from the IP.

\subsection{The trigger and data acquisition system}

ZEUS has a three-stage trigger. The calorimeter first level trigger is critical for this analysis. It uses cells grouped into trigger towers, each approximately 20 $\mathrm{cm} \times 40 \mathrm{~cm}$ in cross-section. For this data a first level trigger was issued if the energy deposited in a trigger tower in any EMC section or in the HAC section of FCAL exceeded a certain threshold. In the FCAL, the EMC threshold was set to $50 \mathrm{GeV}$ for the innermost ring of trigger towers (approximately $20 \mathrm{~cm}$ wide) around the beam pipe, $20 \mathrm{GeV}$ for the second ring 
and $10 \mathrm{GeV}$ elsewhere. The FCAL HAC thresholds were 70.25 and $10 \mathrm{GeV}$ respectively. For the BCAL EMC the threshold was set to $2.5 \mathrm{GeV}$. For the RCAL EMC, the setting was $10 \mathrm{GeV}$ for the innermost ring and $2.5 \mathrm{GeV}$ clsewhere. Events with a signal in the $\mathrm{C} 5$ counter in time with the proton beam were rejected.

Calorimeter signal times, $t_{\mathrm{F}}$ and $t_{\mathrm{R}}$, were defined from groups of FCAL and RCAL cells close to the beam pipe. The time offsets were adjusted so that $t_{F}$ and $t_{\mathrm{R}}$ were approximately zero. Events were rejected in the third level trigger if the following conditions were satisfied:

- at least two photomultiplier channels in FCAL each registered more than $1 \mathrm{GeV}$,

- at least two photomultiplier channels in RCAL cach registered more than $1 \mathrm{GeV}$,

- the signal times were consistent with an interaction upstream of RCAL, i.e., $t_{\mathrm{F}} \sim 0$ ns and $t_{\mathrm{R}} \sim-11 \mathrm{~ns}$.

\section{Data selection criteria}

The triggered events were passed through a filter program requiring

- no veto signal from the VETOWALL counter,

- an EMC trigger signal from the RCAL or BCAL,

- the average time measured in both FCAL and RCAL, when available, as well as their difference, to be within $\pm 6 \mathrm{~ns}$,

- either more than $10 \mathrm{GeV}$ deposited in the FCAL and more than $2.5 \mathrm{GeV}$ deposited in RCAL (condition 1 ),

or more than $20 \mathrm{GeV}$ total energy and $10 \mathrm{GeV}$ transverse encrgy deposited in the whole calorimeter (condition 2).

This filter reduced approximatcly one million triggers to 7087 candidate events. A visual scan of a subsample of these events revealed that beam-gas triggers still dominated. This could also be seen from the $z$ distribution of the reconstructed vertex, which was broad and not centred at zero. In a second filtering, cosmic rays and beam halo muons were rejected with a suitable algorithm and, in order to reduce the beam-gas contamination further, a more refined timing analysis was performed by lowering the threshold for measuring the time to $300 \mathrm{MeV}$ per cell. Furthermore, for events satisfying condition 2 we required that $E_{\mathrm{tol}}-p_{z} \geqslant 12 \mathrm{GeV}^{\# 3}$. This cut discriminates against beam-gas events where almost all of the energy is deposited near the beam pipe in the FCAL.

To ensure that the sample did not contain neutral current ${ }^{* 4}$ DIS events, we searched for scattered electron candidates by using the pattern of energy distribution in the calorimeter. The efficiency for identifying isolated electrons was greater than $99 \%$ for electron encrgies greater than $4 \mathrm{GeV}$. If an electron candidate was found in the calorimeter, $y$ was measured using the rclation

$y_{e}=1-\frac{E_{e}^{\prime}}{E_{e}} \frac{1+\cos \theta_{e}^{\prime}}{2}$

where $E_{\varepsilon}\left(E_{\varepsilon}^{\prime}\right)$ denotes the incident (scattered) electron energy. This relation is valid for colliding beams in the zero mass approximation. Events with $y_{e} \leqslant 0.7$ were rejected. This cut was chosen after detailed Monte Carlo studies for both DIS generated events and photoproduction cvents. It removed approximately 500 events due to DIS, while keeping events where the observed electromagnetic energy deposition is due to photons mainly coming from decays of photoproduced $\pi^{0}$ and $\eta$ mesons. Since this procedure effectively removes scattered electrons in the RCAL, the resulting sample is constrained to $Q^{2}$ values below $4 \mathrm{GeV}^{2}$. We estimate the remaining contamination due to DIS to be below $2 \%$. After these cuts 576 events remained.

The events were examined on visual displays by three independent groups of physicists, revealing that the sample was free from cosmic rays and halo muons. The beam-gas contamination was estimated to be at most $3 \%$. This was confirmed by an independent method not based on visual inspection. The tracking information was used to reconstruct a vertex in $z$. For the $92 \%$ of the events with a reconstructed vertex, the distribution is centred at $-2 \mathrm{~cm}$ with a width of 24 $\mathrm{cm}$. This is in agrcement with MC studies for the efficiency of vertex reconstruction and with the beam bunch lengths as measured by the $\mathrm{C} 5$ counter. Moreover, out of 372 events in those runs with a missing electron bunch, we find the distribution of the bunch

\#3 We define $E_{10 t}$ as the total encrgy deposited in the calorimeter and $p_{z}$ as $\sum E_{l} \cos \theta_{i}$, the sum running over all the calorimeter cells.

*4 The number of charged current events expected with our integrated luminosity is negligible. 
crossing number to be constant for bunch crossing numbers $0-8$ while there was only one event with bunch crossing number 9 , corresponding to the proton unpaired bunch.

\section{The Monte Carlo simulation}

In order to model the expected hadronic final states, we have used the generators PYTHIA [17] and HERWIG [18]. The photon structure function was parametrized according to the prescription of Drees and Grassie [19], while for the proton structure function Morfin-Tung set B1 [20] was used. Extensive Monte Carlo simulations were done varying the $p_{\mathrm{tmin}}$ of the outgoing partons in the hard two-body scattering between 1.0 and $5 \mathrm{GeV}$.

Although the cross-section estimates are not reliable for $p_{\text {tmin }} \leqslant 1 \mathrm{GeV}$, where the validity of perturbation theory is doubtful, it is expected that these models will provide a reasonable description of the hadronic final state at high transverse energy $\left(E_{\mathrm{T}}\right)$ values. These Monte Carlo generators used the Weizsäcker-Williams approximation at the lepton vertex which was constrained to $Q^{2}=0$. This approximation has been estimated to be good to $\sim 10 \%$ under the conditions of the present measurement [21]. The generators take into account the cffect of initial state bremsstrahlung [22]. PYTHIA allows the study of diffractive processes as well as soft $e p$ interactions using a two-string fragmentation picture. The diffractive reactions were generated according to the behaviour measured in low energy photoproduction [23].

In order to estimate the fraction of events where the scattered electron is tagged in the luminosity monitor, we used a modified simulation, generating the scattered electron according to the ALLM [24] prescription, which incorporates a continuous parametrization from the DIS region down to $Q^{2}=0$.

\section{Results}

We now turn to the characteristics of the event sample selected by the above cuts. First we show that the data are consistent with emanating from photoproduction processes. Then we show that the transverse energy distribution cannot be explained just by soft photoproduction processes, but rather requires hard two-body scattering. We then calculate the differential and total cross-sections. We demonstrate that a subset of the data shows clear evidence for two-jet structure back-to-back in the transverse plane. Finally we show that a subset of the two-jet events have energy in the rear (electron) direction, and thus can be interpreted as resolved photon interactions.

\subsection{Kinematic region of accepted events}

We can confirm that the data originate from the photoproduction $\left(Q^{2} \sim 0\right)$ regime by examining the subset of events where the scattered electron is observed in the electron calorimeter used to measure the luminosity. In fact, 96 of the 576 events are tagged in the luminosity monitor with an electron in the energy interval $5 \leqslant E_{e}^{\prime} \leqslant 22 \mathrm{GeV}\left(Q^{2} \leqslant 0.02\right)$, in agreement with Monte Carlo expectations which estimate this fraction to be $20 \%$.

From these tagged events the $\gamma p$ centre of mass energy $W$ was calculated both from the electron energy and from the hadronic system. The results are shown in fig. $2 \mathrm{a}$. The $W$ values calculated from the hadronic system are well correlated with those calculated from the tagged electron, in agreement with detector simulations. Fig. $2 \mathrm{~b}$ shows the distribution in $W$ for the complete sample of events (solid histogram) as 'well as that corresponding to events with an electron observed in the luminosity monitor (dashed histogram). The latter is narrower, reflecting the restricted electron energy acceptance in the luminosity monitor. There is good agreement with Monte Carlo expectations, HERWIG with $p_{\mathrm{tmin}}=1.5 \mathrm{GeV}$, as shown by the dotted curve in fig. $2 b$. In contrast, the $W$ distribution for DIS events has a completely different shape, peaking sharply at small $W[25]$. Thus we conclude that the full sample of events is due to quasi-real $\gamma p$ collisions at a centre of mass energy between $100 \mathrm{GeV}$ and the kinematic limit of $295 \mathrm{GeV}$, the mean value being close to $200 \mathrm{GeV}$. The corresponding acceptance in $y=\left(E_{e}-E_{e}^{\prime}\right) / E_{e}$ is in the range 0.1 to 1 .

\subsection{General characteristics of the event sample}

Figs. $2 \mathrm{c}$ and $2 \mathrm{~d}$ show the total transverse energy and the missing transverse momentum, $p_{1}$. The overall $p_{t}$ 

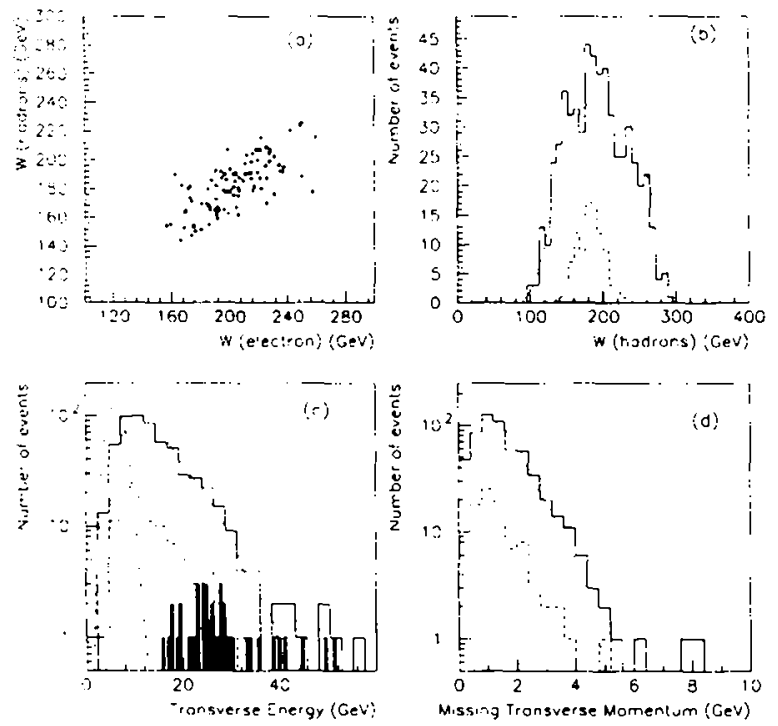

Fig. 2. (a) Correlation between the $\gamma p$ centre of mass energy $(W)$ computed from the hadronic system and from the scattered electron for those events having an electron in the luminosity monitor. (b) The distribution in $W$ calculated from the hadronic system for all events (solid line histogram) and for events with an electron in the luminosity monitor (dashed line histogram). The latter spans a smaller range because of the restricted energy range of the detected electrons. The curve shows the Monte Carlo expectations. (c) Total transverse energy distribution for all events (solid line histogram), for events with an electron tag in the luminosity monitor (dashed line histogram) and for events with two-jet structure (black area). The dashed curve shows the expectations from the HERWIG Monte Carlo generator including direct and resolved photon contributions with $p_{\text {tmin }}=1.5 \mathrm{GeV}$. The dash-dotted curve shows the transverse energy distribution for soft $y p$ interactions. (d) Missing transverse momentum distribution for all events (solid line) and for events with an electron in the luminosity monitor (dashed). The dotted curve shows the Monte Carlo expectations.

is well balanced, as expected for photoproduction interactions, the average $\left\langle\phi_{1}\right\rangle$ being approximately 1.5 $\mathrm{GeV}$. The $E_{\mathrm{T}}$ distribution exhibits, by contrast, a tail that extends beyond $20 \mathrm{GeV}$. This is greatly in excess of what is expected for DIS, where the small transverse momentum carried by the scattered electron is balanced by a single jet. It is also much larger than that expected from soft $\gamma p$ interactions, the tail of which extends to a maximum of about $10 \mathrm{GeV}$ in $E_{\mathrm{T}}$, as shown by the dash-dotted curve in fig $2 \mathrm{c}$. The natural explanation for this is hard scattering between the photon or its constituents, and the proton. The inclusion of such processes in Monte Carlo generators such as PYTHIA and HERWIG reproduces the general features of the data, in particular the high $E_{\mathrm{T}}$ tail, as shown by the dotted curve in fig. $2 c$, which represents HERWIG with $p_{\text {tmin }}=1.5 \mathrm{GeV}$. We note that the events with an electron tag in the luminosity monitor, shown as the dashed histograms in figs. $2 \mathrm{~b}-2 \mathrm{~d}$, exhibit the same behaviour as that characteristic of the total data sample, in particular the high $E_{\mathrm{T}}$ tail.

\subsection{Cross section calculation}

In order to calculate the cross-section for the sample, the trigger and detector acceptances were estimated using Monte Carlo techniques. The overall acceptance varies between $4 \%$ for PYTHIA and $5 \%$ for HERWIG, mainly determined by the trigger. With increasing $E_{\mathrm{T}}$ the acceptance grows and becomes less model dependent, reaching $50 \%$ for $E_{\mathrm{T}}$ values of 40 $\mathrm{GeV}$ [26]. We have restricted ourselves to the region $E_{\mathrm{T}} \geqslant 10 \mathrm{GeV}$ because this region is free from contamination by soft processes, and Monte Carlo calculations that include hard two-body scattering describe the $E_{\mathrm{T}}$ spectrum, as seen in fig. 2 c. We estimate a $20 \%$ systematic error in the acceptance calculation. The integrated luminosity is $2.2 \mathrm{nb}^{-1}$, with a systematic uncertainty of approximately $20 \%$. The total $e p$ crosssection for a transverse energy larger than a given $E_{\mathrm{T}}$ value is shown in fig. 3a. The cross-section exhibits a steep dependence on $E_{\mathrm{T}}$.

There are 391 events with $E_{\mathrm{T}}$ larger than $10 \mathrm{GeV}$ yielding an electroproduction cross-section of $2.4 \pm$ $0.1 \pm 0.7 \mu \mathrm{b}$. Radiative corrections in our kinematic region, where $W$ is larger than $100 \mathrm{GeV}$, are expected to be negligible [11]. The total $e p$ cross-section for $\gamma p$ interactions at HERA has been measured to be $154 \pm$ $16 \pm 32 \mu \mathrm{b}$ [11], which translates to approximately $11 \mu \mathrm{b}$ for electroproduction in the accepted kinematic region $Q^{2} \leqslant 4 \mathrm{GeV}^{2}, y \geqslant 0.1$ [27]. We conclude that approximately $20 \%$ of the total cross-section within this range can be ascribed to hard photoproduction as defined above. In fig. 3a we show the cross-section predicted by HERWIG with a $p_{t \min }$ value of $1.5 \mathrm{GeV}$. The agreement is fairly good. In contrast, calculations with only BGF and QCD Compton processes, i.e., neglecting the resolved photon contribution, give an 

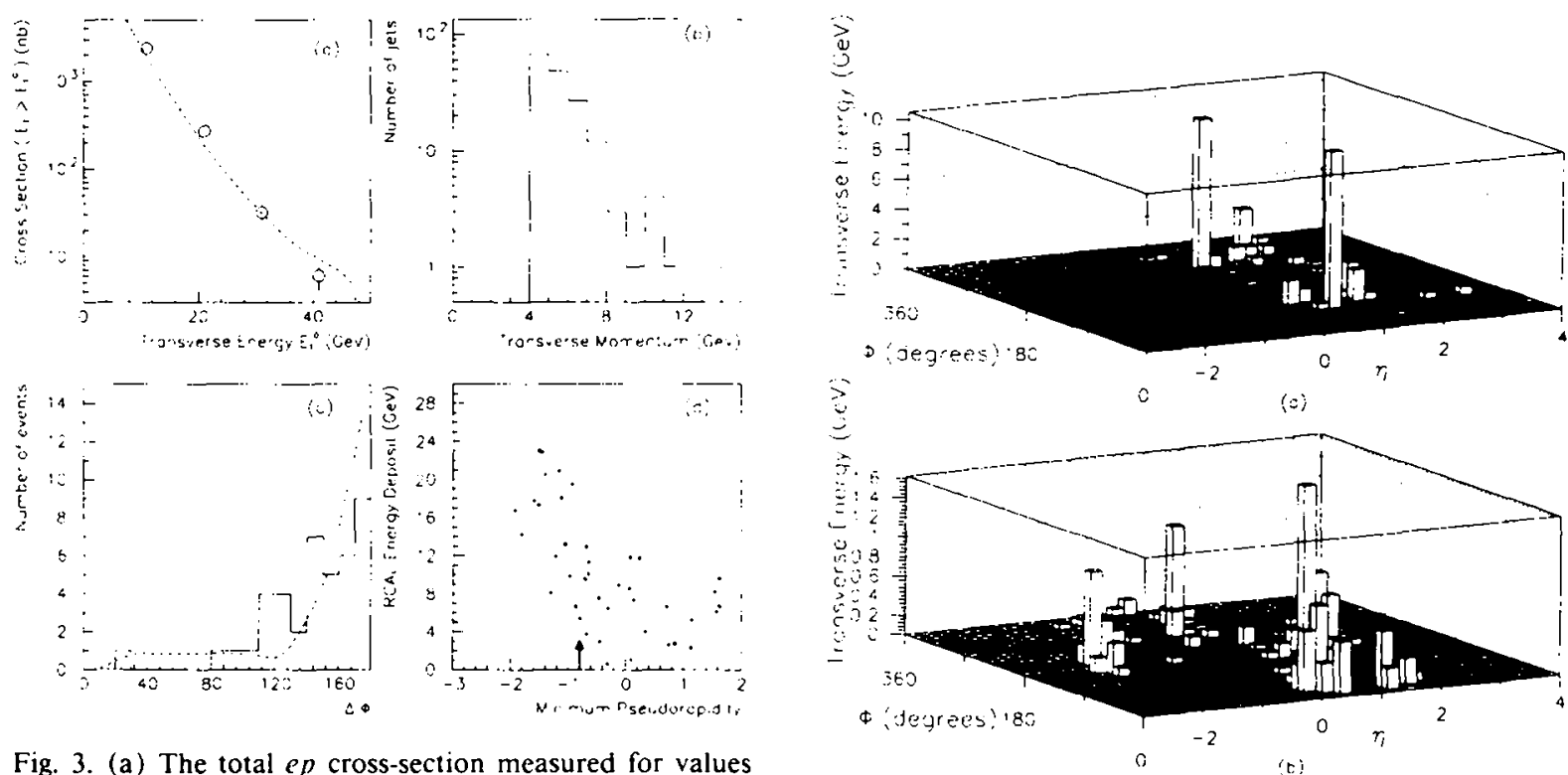

Fig. 3. (a) The total $e p$ cross-section measured for values larger than a given transverse energy $E_{\mathrm{T}}^{0}$. The curve is the predicted cross-section in HERWIG with $p_{\text {tmin }}=1.5 \mathrm{GeV}$, including both direct and resolved photon contributions. (b) Transverse momentum distribution of the identified jets. (c) Distribution of the difference in azimuth between the jets for those events belonging to the two-jet category. The dotted curve represents the Monte Carlo expectations. (d) Correlation between energy deposited in the RCAL versus the minimum pseudorapidity of the two jets in the two-jet sample. The arrow marks the RCAL-BCAL boundary.

electroproduction cross-scction which is at least an order of magnitude smaller.

\subsection{Evidence for jet structure}

The observation of a large $E_{\mathrm{T}}$ tail in the data as well as the measurement of the total cross-section lend support to the hypothesis that our high $E_{\mathrm{T}}$ data includes a large component of hard collisions. If this is indeed the case there should be a two-jet structure in a subset of the data. We searched for such final states using a jet finding algorithm in pseudorapidity $(\eta)-$ azimuth $(\phi)$ space based on the Snowmass convention [28]. The cone radius $R=\left(\Delta \phi^{2}+\Delta \eta^{2}\right)^{1 / 2}$ in the algorithm was set to 1 unit and cells with EMC (HAC) transverse energy below $50 \mathrm{MeV}$ ( $100 \mathrm{MeV}$ ) were excluded. In order to ensure that the results are not biased by fragments from the proton remnant, whose fragmentation properties at these cnergies are not well

Fig. 4. (a) Transverse cnergy in $\eta-\phi$ space ( $\eta$ stands for pseudorapidity and $\phi$ for azimuth angle) for an event with a two-jet structure. (b) Transverse energy in $\eta-\phi$ space for an cvent showing a two-jet structure plus additional activity in the incident electron direction associated with the photon remnant.

determined, we cxcluded cells with a polar angle less than $10^{\circ}$. Preclusters were formed around cells with $E_{\mathrm{T}}$ larger than $300 \mathrm{MeV}$, and the final clusters were called jets if their transverse momentum was larger than $4 \mathrm{GeV}$ and $\eta \leqslant 2$, i.e., polar angles larger than $15^{\circ}$.

It is found that 7\% $(0.8 \%)$ of the events are of twojet (three-jet) type, in good agrecment with Monte Carlo calculations predicting $8 \%(0.7 \%)$. Fig. 4a shows the transverse encrgy distribution in the $\eta, \phi$ plane of one of the two-jet events. Two well collimated hadron jets with energies greater than $5 \mathrm{GeV}$ are seen, their separation in $\phi$ being close to $180^{\circ}$. In fig. $3 \mathrm{~b}$ we show the $p_{\mathrm{t}}$ distribution of all of the observed jets and in fig. $3 c$ the difference in azimuth between the jets in the two-jet sample. A peak around $180^{\circ}$ is observed, showing evidence that the two-jet sample is dominated by back to back jets in the transverse plane. Furthermore, 7 of the 41 two-jet events have an electron in the luminosity monitor, in agreement with the Monte Carlo expectation of 8 events.

Although the limited statistics preclude an analy- 
sis of the jet activity as a function of the total transverse energ:. We shou in fig. 2c ldashed areal the iransicerse energy distribution for events with a tu(t)jet structure. This clearly shows that these events oc. cur at the higher values of $F_{\text {. }}$, as expected, and as is obsericd in hadron-hadion colfusions $\{24,30\}$

\subsection{Evidence for remnants of the resolved photon}

As discussed above, a major fraction of the high $E_{\mathrm{T}}$ events is expected to come from resolved photon interactions. Long range forces during the hadronization should cause the fragments of the photon remnant to leave the beam pipe and make their observation possible [31].

Fig. 3d shows the energy deposited in RCAL versus the minimum pseudorapidity of cither of the two jets for our two-jet sample. If direct photon interactions were the sole origin of these events. substantial energy in RCAL would be expected for events with the minimum jet pseudorapidity $\left(\eta_{\min }\right)<-1$, falling to cssentially zero as the jets become more distant from the $\mathrm{RCAL}$ region $\left(\eta_{\mathrm{m} i \mathrm{n}}>0\right)$ ). This trend is indeed observed in fig. 3d. However, in addition the data show a sizeable energy detected in RCAL, 4 to $8 \mathrm{GeV}$, even when both jets are far from the RCAL itself, the nearest jet being as much as three units of rapidity away. These events are therefore interpreted as originating from the resolved photon process, where the two parton jets go forward and the photon remnant go approximately in the direction of the incident electron, which is close to the direction of the virtual photon.

By requiring $\eta_{\min }>0$ and RCAL energy larger than $4 \mathrm{GeV}$ for the two-jet sample, we select 11 resolved photon candidates, in agreement with Monte Carlo calculations which predict 16 events in this region. Onc of the resolved photon candidates is shown in fig. 4b. There is substantial energy in the RCAL with a large pseudorapidity gap between it and the two jets. For the resolved photon events we can estimate the fractional momenta of the interacting partons by several methods, for example using the invariant masses of both the jet-jet system and the jet-jet-remnant system, as described in ref. [31]. We have checked by Monte Carlo methods that, even with the low encrgy jets characteristic of this analysis, there is satisfactory accuracy in the reconstruction of the parton fractional momenta. For the four events in the sam- ple in which the scattered electron is detected in the luminosily monitor. the parton fractional momenta can be measured using only the jet energies and the tagged electron energ: In these events the observed RC:IL energ: is in accord with the prediction from the reconstructed parton hinematicis.

\section{Summary and conclusions}

We have isolated a sample of photoproduction events at HERA that are well balanced in $p_{\mathrm{l}}$. The distribution in transverse energy exhibits a tail extending up to $40 \mathrm{GeV}$, which cannot be explained either by soft processes or by a current jet balancing the $p_{\imath}$ of a scattered electron. Clear back-to-back twojet events are found in a region of phase space well scparated from the proton fragments.

The rates and cross-section are in agreement with Monte Carlo models containing resolved photon processes. A subset of the events with two-jet structure exhibits energy in the rear direction consistent with expectations for the fragments of the photon. These properties are also shown by that subset of the data in which the scattered electron is tagged at small angles in the luminosity detector. These effects are evidence for hard two-body scattering between partons in the photon and the proton.

\section{Acknowledgement}

The strong support and encouragement by the DESY Directorate Professor V. Soergel, Dr. H. F. Hoffmann, Dr. H. Krech. Dr. J. May, Dr. D.M. Polter, Professor P. Söding. Professor G.A. Voss, and Professor A. Wagner have been invaluable, as well as the support by Dr. G. Söhngen.

The experiment was made possible by the inventiveness and the diligent efforts of the HERA machine group who succeeded in making HERA run in a very short time.

The design. construction, and installation of the ZEUS detector has been made possible by the ingenuity and dedicated effort of many people from inside DESY and from the home institutes who are not listed as authors. Their contributions are acknowledged with great appreciation. 
This work has been supported by the Natural Sciences and Engineering Research Council, and the FCAR of Quebec, Canada, by the German Federal Ministry for Research and Technology (BMFT), by the Deutsche Forschungsgemeinschaft (DFG), by the Italian National Institute for Nuclear Physics (INFN), by the Japanese Ministry of Education, Science and Culture (the Monbusho) and its grants for Scientific Research, by the Netherlands Foundation for Research on Matter (FOM), by the Polish Government and Ministry of Education Research Programs, by the Spanish Ministry of Education and Science through funds provided by CICYT, by the UK Science and Engineering Research Council, by the MINERVA Foundation, by the Israel Academy of Science and the Israeli Department of Energy, by the German Israeti Foundation, by the US Department of Energy and by the US National Science Foundation.

\section{References}

[1] See A. Ali et al., Proc. HERA Workshop (DESY, 1987) p. 395.

[2] See S.J. de Jong et al., Proc. HERA Workshop (DESY, 1987) 533.

[3] E. Witten, Nucl. Phys. B 120 (1977) 189.

[4] W.J. Stirling and Z. Kunszt, in: Proc. HERA Workshop (DESY, 1987) p. 331;

M. Drees and R.M. Godbole, Phys. Rev. D 39 (1989) 169.

[5] AMY Collab., R. Tanaka et al., Phys. Lett. B 277 (1992) 215.

[6] NA1 4 Collab., E. Auge et al., Phys. Lett. B 168 (1986) 163; B 182 (1986) 409;

NA14 Collab., R. Barate et al., Phys. Lett. B 174 (1986) 458.

[7] European Muon Collab., J.J. Aubert et al., Phys. Lett. B 100 (1981) 433; European Muon Collab., M. Arneodo et al., Z. Phys. C 36 (1987) 527.

[8] Wa69 Collab., R.J. Apsimon et al., Z. Phys. C 43 (1989) 63; C 46 (1990) 35.

[9] F. Eisele, talk Intern. Conf. on High energy physics (Dallas, August 1992).

(10) The ZEUS Detector, Status report (1989), DESY PRC 89-01.
[11] ZEUS Collab., M. Derrick et al., Phys. Lett. B 293 (.1992) 465 .

[12] C.B. Brooks et al., Nucl. Instrum. Methods A 283 (1989) 477.

[13] ZELS Calorimeter Group, A. Andresen et al., Nucl. Instrum. Methods A 309 (1991) 101.

[14] A. Bernstein et al., Nucl. Instrum. Methods, to be submitted;

U. Mallik et al., Proc. Intern. Conf. on Calorimetry in High energy physics (FNAL, 1990).

[15] A. Caldwell et al., Nucl. Instrum. Methods A 321 (1992) 352;

see also A. Caldwell, talk Intern. Conf. on High energy physics (Dallas, August 1992);

L. Hervas, thesis, Universidad Autonoma de Madrid, unpublished.

[16] J. Andruszków et al., DESY 92-066 (1992).

[17] H.-U. Bengtsson and T. Sjöstrand, Comput. Phys. Commun. 46 (1987) 43; T. Sjostrand, CERN-TH.6488/92.

[18] G. Marchesini et al., Comput. Phys. Commun. 67 (1992) 465.

[19] M. Drees and K. Grassie, Z. Phys. C 28 (1985) 451.

[20] J.G. Morfin and W.K. Tung, Z. Phys. C 52 (1991) 13.

[21] A.I. Lebedev, Proc. Workshop on Physics at HERA (DESY, 1991) p. 613.

[22] R. Kleiss et al., in: $Z^{0}$ physics at LEP, eds. G. Altarelli, R. Kleiss and G. Verzegnassi, CERN 89-08, Vol. 3, p. 1.

[23] K. Goulianos, Phys. Rep. 101 (1983) 169.

[24] H. Abramowicz, E. Levin, A. Levy and U. Maor, Phys. Lett. B 269 (1991) 465 .

[25] B. Loehr, talk Intern. Conf. on High energy physics (Dallas, August 1992).

[26] J.F. de Trocóniz, Ph.D. thesis Universidad Autónoma de Madrid, to be presented.

[27] E. Lohrmann, Proc. Workshop on HERA physics (DESY, 1992) p. 519.

[28] J. Huth et al., FERMILAB-Conf-90-249-E.

[29] UA2 Collab., J. Alitti et al., Phys. Lett. B 257 (1991) 232; UA2 Collab., J.A. Appel et al., Phys. Lett. B 160 (1985) 349.

[30] UAl Collab., C. Albajar et al., Nucl. Phys. B 309 (1988) 405.

[31] G. D'Agostini and D. Monaldi, Proc. Workshop on HERA physics (DESY, 1992) p. 527. 